\title{
Penser la fin de vie
}

\section{Jean Martin}

Dr méd., membre de la rédaction

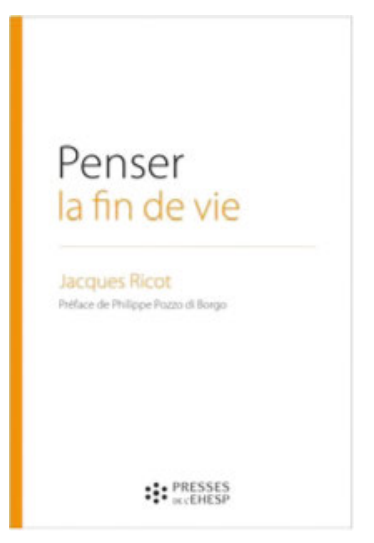

Jacques Ricot

Penser la fin de vie

Rennes: Presses de

l'EHESP (Ecole des

Hautes Etudes en Santé

Publique); 2017.

347 p. 29.60 CHF.

Jacques Ricot a enseigné la philosophie à l'Université de Nantes et est aussi impliqué dans la formation de professionels de santé. Il a écrit plusieurs livres en rapport avec la fin de vie, l'euthanasie, le suicide. Son dernier ouvrage est une somme différenciée, sur des thèmes qui vont de la condition humaine à la déontologie et la pratique médicales - y compris obstination déraisonnable, lutte contre la douleur, soins palliatifs, tension entre bienveillance du soignant et droits du patient. Décrivant entre autres les modèles français et britannique de l'autonomie. Ses derniers chapitres traitent de la «tentation de l'euthanasie» et de la notion débattue et polysémique de dignité. A ces sujets, on laisse entendre de manière à vrai dire discutable que ceux qui admettent l'euthanasie voudraient en faire un droit opposable (à savoir qu'on pourrait exiger de l'Etat qu'il s'engage à la rendre possible, dans chaque situation - ce qui n'est évidemment pas le cas: cela resterait un accord entre "partenaires» d'accord d'y procéder).

\section{La France et d'autres pays}

Le propos de Ricot reste marqué par les positions majoritairement (ré)affirmées ces dernières décennies en France, mais on doit apprécier le réel intérêt et le respect qu'il montre (bien qu'il ne puisse s'y rallier et qu'elles le laissent perplexe) pour les opinions et déterminations différentes dans d'autres pays et cultures généralement considérés comme civilisés et/mais qui acceptent l'assistance au suicide - Suisse - et même l'euthanasie - Benelux. A noter que l'auteur s'éloigne dans une large mesure de ce qui peut rester paterna- liste dans la pratique médicale, en se ralliant à la déontologie renouvelée maintenant fermement établie dans les textes. Ricot rappelle que le secret médical n'est - bien sûr - jamais opposable au patient. Il faut aussi affirmer que «mentir n'est jamais justifié ni éthiquement, ni thérapeutiquement» - dans un passé encore récent toutefois, on prenait des libertés avec ces règles majeures... Est évoqué dans ce contexte un texte connu de 1950 de Louis Portes, alors président de l'Ordre national des médecins, dont Ricot dit: «Le paternalisme qui suinte à travers les expressions utilisées nous est devenu inaudible, l'inégalité qui s'affiche sans fard, au prix d'une infantilisation du malade..." Dans un autre registre et sous le titre «Les méfaits du dolorisme», il écrit: "Les médecins ont parfois pensé que l'héroïsme du patient face à la douleur lui octroyait une énergie efficace [...] Ces théories, jamais validées, ont vécu.»

\section{Autonomie(s)}

«La France offre un modèle, presque chimiquement pur, d'une préférence pour un régime de protection des individus contre les dérives éventuellement nuisibles de l'exercice de leurs libertés.» Citant Suzanne Rameix: «L'Etat protège contre eux-mêmes les citoyens qui porteraient attente à l'intégrité de leur corps ou à leur vie.»

\section{Son dernier ouvrage est une somme différen-} ciée, sur des thèmes qui vont de la condition humaine à la déontologie et la pratique médicales.

Ricot: "L'autonomie est la soumission à une loi rationnelle universalisable» (chassez le paternalisme, il revient au galop, diront certains hors de France). Plus loin: «Pour l'essentiel, dans le contexte culturel anglo-saxon, on privilégie l'indépendance de l'individu par rapport à la soumission à une loi [...], une auto-détermination dont la seule limite serait la non-malfaisance.» Aussi: «La logique de la relation médicale peut alors se décliner de façon contractuelle», contrairement à la vue française des devoirs fondamentaux du soignant, parfois contre l'avis du malade.

«En dernière instance, qui juge du caractère utile du traitement? C'est le patient, dont la volonté doit être 
respectée [...] Le législateur a voulu ajouter deux clauses: d'une part, l'arrêt des traitements ne signe pas la fin du pacte de soin; d'autre part, le médecin doit vérifier que la décision du patient a bien été réitérée, en raison des conséquences éventuellement irrémédiables.»

\section{Consentement et directives anticipées vs bienveillance}

«L'insistance sur le recueil du consentement et l'irruption des notions de personne de confiance et de directives anticipées dans la législation sont des indices très éloquents de la modification de la relation qui voit le devoir de bienfaisance du médecin reculer devant le respect de l'autonomie du patient.» Correct. Toutefois, Ricot reste ambivalent: «Le soignant pourrait devenir

\section{Ricot rappelle que le secret médical n'est - bien sûr-jamais opposable au patient.}

l'instrument passif d'une volonté toute-puissante, celle du malade.» Plus loin: «La question aiguë posée par le mouvement impétueux de la modernité est ailleurs. C'est celle de la liberté de quitter la relation de soin pour anticiper la mort qui s'annonce.» Des craintes donc. Frilosités?

Pourtant, dans la préface de l'ouvrage, le Dr Jean Leonetti, rapporteur de la loi qui porte son nom, écrit: «Les auteurs et les témoins du soin ne peuvent que souscrire à cette phrase [de Ricot dans un autre livre]: 'La compassion, dissociée de l'exercice rationnel, ne saurait être promue au rang de repère éthique, en particulier pour les situations de fin de vie.'»

\section{Intentionnalité}

Sur cette question, jugée fondamentale en France, Ricot cite J.-C. Fondras et S. Rameix: "Comment juger si une sédation profonde, continue jusqu'au décès, est sédative ou euthanasique? [...] Devant des effets secon- daires problématiques (abréger la vie du patient ou porter atteinte à son autonomie), la détermination de la véritable intention et de sa légitimité reste un point crucial.» C'est là qu'on peut ou même doit diverger. En effet, si la remarque ci-dessus est impeccable théoriquement, il est illusoire pratiquement/cliniquement de vouloir en faire un critère déterminant aujourd'hui dans les décisions prises au lit du malade en fin de vie qu'on rappelle par exemple que plus de trois quarts des décès survenant en service de soins intensifs font suite à une décision médicale. Dans de nombreuses situations, se fixer sur une appréciation de l'intentionnalité n'est pas vraiment pertinent, en tout cas terriblement subjectif - qu'il s'agisse de l'acteur ou de l'observateur; et dite appréciation n'est pas possible de la manière précise que voudraient les tenants de ce critère. De plus, on a le droit ici de mettre en relation les déclarations de principes présentés comme absolument non négociables et le fait que personne ne conteste qu'il y a en France, bon an, mal an, un nombre certain d'euthanasies qui ne disent pas leur nom. L'auteur de cette recension dit sa difficulté devant des positions qui décident d'occulter une partie de la réalité pourtant tolérée, pour ne pas dire admise, par la société.

\section{L'auteur de cette recension dit sa difficulté devant des positions qui décident d'occulter une partie de la réalité pourtant tolérée, pour ne pas dire admise, par la société.}

Ricot ne souhaite pas qu'une confrontation stérile perdure entre ces attitudes françaises (dont il reconnaît au reste qu'elles sont actuellement indéniablement bousculées, y compris parmi la population) et celles de pays libéraux (anglo-saxons, d'autres aussi). Il préférerait qu'on aille vers un dialogue; s'il se fait sur des bases objectives, c'est une direction qu'on peut certainement saluer. 